\title{
Coping with Outside-the-Box Attacks
}

\author{
Edward W. Felten \\ Department of Computer Science \\ and \\ Woodrow Wilson School of Public and International Affairs \\ Princeton University
}

There is a long history of security attacks that succeed by violating the system designer's assumptions about how things work. Even if a designer does everything right - within the "obvious" model — such attacks can still succeed. How can we, as designers and verifiers of systems, cope with these "outside-the-box" attacks?

The classic examples of assumption-violating attacks are the timing attacks on cryptosystems first introduced by Kocher [1. Cryptosystems are designed so that an attacker who has black-box access to an implementation (and does not know the secret key) cannot deduce the key. Extensive mathematical analysis of the input-output behavior of cryptographic functions led to the belief (though unfortunately not proof) that an attacker who can observe the input-output behavior of cryptosystems cannot feasibly find the secret key. Kocher showed that even if this is true, the running time of common cryptographic algorithms does depend on the secret key. Though the dependence of running time on the key is complex, Kocher showed how to use randomized experiments to extract enough signal to deduce the key, at least in principle. Brumley and Boneh later showed that such attacks are practical, even across a network[2].

Systems can be redesigned to resist Kocher-style timing attacks, and many systems were redesigned after the attacks became known. However, any new attack that breaks existing systems imposes significant cost and puts existing systems at risk. Designers began to worry that more outside-the-box attacks might be coming.

This fear turned out to be justified, as more attacks on cryptosystems were uncovered. Kocher, Jaffe, and Jun discovered that the power consumption of crypto implementations leaked information about their internal computations, allowing secret keys to be discovered. Fine-grained fluctuations in power consumption during a computation turned out to leak even more information [3]. Again, systems could be redesigned - though not so easily this time - but again existing systems were vulnerable.

Cryptosystems fell victim to other outside-the-box attacks. Boneh, DeMillo, and Lipton showed how to defeat public-key cryptosystems by inducing faults in the cryptographic computation. Remarkably, even if the attacker couldn't determine exactly where, when, and how the error occurred, just knowing there was an error and having access to the corrupted output was sufficient to deduce the secret key in most cases [4. Biham and Shamir found a similar result for

A. Gupta and S. Malik (Eds.): CAV 2008, LNCS 5123, pp. 3-4, 2008.

(C) Springer-Verlag Berlin Heidelberg 2008 
symmetric cryptosystems [5]. More systems had to be redesigned, even though they were secure under (then-)existing models.

Similar attacks worked in non-crypto settings as well. Kuhn and Anderson showed attacks on satellite television smartcards [6]. Govindavajhala and Appel showed how to break Java virtual machine security by using heatlamps to generate thermal errors in PC execution [7. Several researchers showed how to breach security barriers by observing the timing of execution. Halderman et al. defeated operating system memory protection by cooling DRAM chips then cutting system power 8 .

The upshot of all this is that outside-the-box attacks seem to be a fact of life. We can expect to see more attacks new unexpected attack modes. Though we can't predict what the attacks will be, we know they are coming. How can we, as system designers and verifiers, cope?

We are often advised to think outside the box. While it is good to widen our horizons and think creatively from time to time, "think outside the box" is not really actionable advice. What we need instead is to find a better box-one whose boundaries are not so often broken.

How exactly to do this is not a question we can answer now. One goal of this presentation is to catalyze a discussion about how we might improve our models, or how we might design systems to be more resilient against failures of our models to match reality. Until we can answer this question, our systems will remain fragile, no matter how hard we work on verifying them.

\section{References}

1. Kocher, P.C.: Timing attacks on implementations of diffie-hellman, rsa, dss, and other systems, 104-113 (1996)

2. Brumley, D., Boneh, D.: Remote timing attacks are practical (1996)

3. Kocher, P., Jaffe, J., Jun, B.: Differential power analysis. In: Wiener, M.J. (ed.) CRYPTO 1999. LNCS, vol. 1666, pp. 388-397. Springer, Heidelberg (1999)

4. Boneh, D., DeMillo, R., Lipton, R.: On the importance of checking cryptographic protocols for faults. Journal of Cryptology 14(2), 101-119 (2001)

5. Biham, E., Shamir, A.: Differential fault analysis of secret key cryptosystems. In: Kaliski Jr., B.S. (ed.) CRYPTO 1997. LNCS, vol. 1294, pp. 513-525. Springer, Heidelberg (1997)

6. Anderson, R., Kuhn, M.: Tamper resistance - a cautionary note. In: Proc. USENIX Workshop on Electronic Commerce, pp. 1-11 (November 1996)

7. Govindavajhala, S., Appel, A.W.: Using memory errors to attack a virtual machine. In: Proc. IEEE Symposium on Security and Privacy (May 2003)

8. Halderman, J.A., Schoen, S.D., Heninger, N., Clarkson, W., Paul, W., Calandrino, J.A., Feldman, A.J., Appelbaum, J., Felten, E.W.: Lest we remember: Cold boot attacks on encryption keys. In: Proc. USENIX Security Symposium (2008) 